\title{
Pulsar timing arrays and the cosmological constant
}

Domènec Espriu

Citation: AIP Conference Proceedings 1606, 86 (2014);

View online: https://doi.org/10.1063/1.4891120

View Table of Contents: http://aip.scitation.org/toc/apc/1606/1

Published by the American Institute of Physics 


\title{
Pulsar Timing Arrays and the cosmological constant
}

\author{
Domènec Espriu \\ Departament d'Estructura i Constituents de la Matèria and Institut de Ciències del Cosmos (ICCUB), \\ Universitat de Barcelona, Martí i Franquès, 1, 08028 Barcelona, Spain.
}

\begin{abstract}
In this talk I review how a non-zero cosmological constant $\Lambda$ affects the propagation of gravitational waves and their detection in pulsar timing arrays (PTA). If $\Lambda \neq 0$ it turns out that waves are anharmonic in cosmological FriedmannRobertson- Walker coordinates and although the amount of anharmonicity is very small it leads to potentially measurable effects. The timing residuals induced by gravitational waves in PTA would show a peculiar angular dependence with a marked enhancement around a particular value of the angle subtended by the source and the pulsars. This angle depends mainly on the actual value of the cosmological constant and the distance to the source. Preliminary estimates indicate that the enhancement can be rather notorious for supermassive black hole mergers and in fact it could facilitate the first direct detection of gravitational waves while at the same time representing a 'local' measurement of $\Lambda$.
\end{abstract}

Keywords: Cosmological constant, dark energy, gravitational waves, pulsar timing arrays

\section{INTRODUCTION}

There is a fair amount of evidence suggesting that the space time where we live is globally de Sitter with a value for the cosmological constant estimated [1] to be around $\Lambda \simeq 10^{-52} \mathrm{~m}^{-2}$. This has obvious effects on cosmology - at very large scales. These effects are of no concern to us here.

Instead we would like to help answer the question: does $\Lambda$ have a 'local' influence?, where local means at moderate values of the redshift $z$. In short, can the cosmological constant be measured 'locally'? This is an important question because it may settle the issue as to whether $\Lambda$ is a truly fundamental property of space-time, a basic constant of nature present at all scales, rather than a way of providing some effective description relevant only at cosmological distances.

A lot of work has been devoted to finding traces of the existence of the cosmological constant at sub-cosmological scales such as in cluster of galaxies [2], so far without a clear conclusion or very relevant bounds. Several works study the effect of $\Lambda$ on the gravitational bending of ligh $[3,4,5]$ or the Shapiro effect [6]. Some authors have even advocated more exotic effects such as providing an explanation the well known Pioneer anomaly[7] or the apparent increse of the astonomical unit with time[8].

Discussions in the literature regarding the above points tend to be confusing. Effects range from surprisingly large to zero. Not surprisingly the source of most discrepancies is the meaning of the different coordinate systems and their physical realization.

Here we propose to try to find 'local' effects of $\Lambda$ by studying the propagation of gravitational waves (GW) in an space endowed with a cosmological constant. This may seem hopeless at first as there is no direct detection of a GW yet, let alone possible modifications due to $\Lambda \neq 0$. However because the nature of the cosmological constant is quite unclear it is plausible that it should be attributed to the gravitational interaction itself - a fundamental property of space-time - and accordingly placed naturally on the 1.h.s. of Einstein equations. Then it seems quite natural to investigate how the fundamental excitations of gravity are modified by $\Lambda$. This issue has been studied in [9].

Has $\Lambda$ possible observational consequences on GW in spite of its currently preferred very small value? We will see that the answer to this question is somewhat surprising. What follows is an extended version of the results found by us and described in [10].

For a previou attempt to find effects of a non-zero cosmological constant using GW see [11].

\section{LINEARIZATION OF THE FIELD EQUATIONS IN THE PRESENCE OF $\Lambda$}

Keeping control of the different orders in $\Lambda$ will be essential to our discussion. This will become obvious in the subsequent.

II Russian-Spanish Congress on Particle and Nuclear Physics at all Scales, Astroparticle Physics and Cosmology AIP Conf. Proc. 1606, 86-98 (2014); doi: 10.1063/1.4891120

(C) 2014 AIP Publishing LLC 978-0-7354-1242-2/ $\$ 30.00$ 
Let us start by linearizing Einstein equations since after all GW are solutions of the linearized equations:

$$
R_{\mu v}-\frac{1}{2} g_{\mu v} R+\Lambda g_{\mu v}=-\kappa T_{\mu v}
$$

In the linearized approximation we assume $g_{\mu \nu}=\eta_{\mu v}+h_{\mu v},\left|h_{\mu v}\right| \ll 1$ and then

$$
R_{\mu v}=\frac{1}{2}\left(\square h_{\mu v}+h_{, \mu v}-h_{\mu, v \lambda}^{\lambda}-h_{v, \mu \lambda}^{\lambda}\right) .
$$

A gauge choice is mandatory to solve these equations. A common choice is to select the Lorenz gauge

$$
\partial_{\mu} h_{v}^{\mu}=\frac{1}{2} \partial_{v} h \leftrightarrow \partial_{\mu} \tilde{h}_{v}^{\mu}=0
$$

where

$$
\tilde{h}_{\mu v}=h_{\mu v}-\frac{1}{2} \eta_{\mu v} h
$$

The field equation in Lorenz gauge are

$$
\square\left(h_{\mu v}-\frac{1}{2} \eta_{\mu v} h\right)+2 \Lambda h_{\mu v}=-2 \Lambda \eta_{\mu v} .
$$

Whether the term of order $\mathscr{O}(h \Lambda)$ needs to be considered or not depends on the relative magnitude of $\square h$ and $\Lambda$. If the $\Lambda h_{\mu v}$ term on the 1.h.s. is omitted (and only in this case) there is a residual gauge freedom within the Lorenz gauge.

$$
x^{\mu} \rightarrow x^{\prime \mu}=x^{\mu}+\xi^{\mu}
$$

as long as $\xi^{\mu}$ is an harmonic function, $\square \xi^{\mu}=0$. All this is of course well known and standard texbook material. If we set $\Lambda$ to zero we get the standard treatment of gravitational waves - in Minkowski space time. It is clear that $\Lambda \neq 0$ will modify the for of the solution by terms of order $\Lambda$. But we also need to know what choice of coordinates the linearization and the Lorenz condition implies, if any. This is not a totally trivial issue in de Sitter.

\section{'GOOD’ AND ‘BAD’ COORDINATE SYSTEMS IN DE SITTER}

Before giving the solution to the field equation in the Lorenz gauge (the one where GW are usually treated) let us discuss several possible coordinate choices in de Sitter space-time. Convenient references are given in [12]. See also [13].

A static metric: Schwarzschild-de Sitter (SdS)

$$
d s^{2}=\left[1-\frac{\Lambda}{3} \hat{r}^{2}\right] d \hat{t}^{2}-\left[1-\frac{\Lambda}{3} \hat{r}^{2}\right]^{-1} \hat{r}^{2}+\hat{r}^{2} d \Omega^{2}
$$

This metric has not quite spherical symmetry.

A position independent metric: Friedmann-Robertson-Walker (FRW)

$$
d s^{2}=d T^{2}-\exp \left(2 \sqrt{\frac{\Lambda}{3}} T\right) d \vec{X}^{2}
$$

This metric incorporates the physical principles of cosmological homogeneity and isotropy. The coordinates $X^{i}$ are comoving coordinates anchored in space that expand with the universe. These are the coordinates where our world appears homogeneous and isotropic.

None of the previous metrics obey the Lorenz gauge condition. Of course there is no reason why they should because they are not solutions of Einstein equations linearized around Minkowski space-time, so in order to match with the discussion in the previous section let us proceed to lineariarize the two metrics. For the SdS metric this is quite easy as the $\mathrm{SdS}$ metric is expandable in integer powers of $\Lambda$

$$
d s^{2} \simeq\left[1-\frac{\Lambda}{3} \hat{r}^{2}\right] d \hat{t}^{2}-\left[1+\frac{\Lambda}{3} \hat{r}^{2}\right] \hat{r}^{2}+\hat{r}^{2} d \Omega^{2} .
$$


It should therefore obey a linearized version of Einstein equations, although as can be easily verified not in the Lorenz gauge.

On the contrary it is clear that the FRW coordinate choice cannot be linearized in $\Lambda$ because it contains odd powers of $\sqrt{\Lambda}$

$$
d s^{2}=d T^{2}-\exp \left(2 \sqrt{\frac{\Lambda}{3}} T\right) d \vec{X}^{2}
$$

and therefore it is impossible that it can fulfill any linearized Einstein equation, even if $t<<1 / \sqrt{\Lambda}$ as it is not expandable in integer powers of $\Lambda$.

One can work out the exact transformation between the two coordinate systems. We shall reserve capital letters for FRW coordinates and the hatted lower case ones for SdS (the reason for the hat will be evident below)

$$
\begin{gathered}
\hat{r}=e^{T \sqrt{\Lambda / 3}} R \\
\hat{t}=\sqrt{\frac{3}{\Lambda}} \log \left(\frac{\sqrt{3}}{\sqrt{3-\Lambda e^{2 T \sqrt{\Lambda / 3} R^{2}}}}\right)+T
\end{gathered}
$$

where $T$ and $R$ are the cosmological time and comoving coordinates whose physical realization is clear. This transformation is valid inside the cosmological horizon, i.e. $R<\frac{1}{\sqrt{\Lambda}}$.

\section{LINEARIZED BACKGROUND AND LINEARIZED GW SOLUTIONS}

Let us now return to Eq. 5. We shall work in the linearized approximation both for the background modification (with respect to flat Minkowski space-time) $h_{\mu \nu}^{\Lambda}$ and for gravitational wave perturbations $h_{\mu \nu}^{W}$. We follow here the discussions in $[9,13]$. The total metric will be written as

$$
g_{\mu v}=\eta_{\mu v}+h_{\mu v}^{\Lambda}+h_{\mu v}^{W}, \quad\left|h_{\mu v}^{\Lambda, W}\right| \ll 1
$$

Let us focus on the background. In the Lorenz gauge and neglecting $\Lambda h_{\mu v}^{\Lambda}$

$$
\square \tilde{h}_{\mu v}=-2 \Lambda \eta_{\mu v}, \quad \partial_{\mu} \tilde{h}_{v}^{\mu}=0,
$$

This has as a particular solution

$$
\tilde{h}_{\mu v}=-\frac{\Lambda}{18}\left(4 x_{\mu} x_{v}-\eta_{\mu \nu} x^{2}\right) \Rightarrow h_{\mu v}=\frac{\Lambda}{9}\left(x_{\mu} x_{v}+2 \eta_{\mu \nu} x^{2}\right)
$$

The general solution is the former plus any solution of $\square \tilde{h}_{\mu \nu}$ but we call the latter 'waves' rather than 'background'. Indeed the equation for GW is particularly simple in Lorenz coordinates if the $\Lambda h_{\mu \nu}^{W}$ term is neglected.

$$
\square \tilde{h}_{\mu v}^{W}=0
$$

i.e. it is truly a wave equation.

Now we have to answer the following question: What is the physical realization of the coordinates $x, t$ where we just solved Einstein equations in the Lorenz gauge?

Let us take advantage of the residual gauge invariance that exists if the term $\Lambda h_{\mu \nu}$ is neglected. Then the solution

$$
\tilde{h}_{\mu v}=-\frac{\Lambda}{18}\left(4 x_{\mu} x_{v}-\eta_{\mu v} x^{2}\right)
$$

or

$$
h_{\mu v}=\frac{\Lambda}{9}\left(x_{\mu} x_{v}+2 \eta_{\mu v} x^{2}\right)
$$

can be transformed into a static metric — still in Lorenz gauge. 
The following change of coordinates

$$
\begin{gathered}
x=x^{\prime}+\frac{\Lambda}{9}\left(-t^{\prime 2}-\frac{x^{\prime 2}}{2}+\frac{\left(y^{\prime 2}+z^{\prime 2}\right)}{4}\right) x^{\prime} \\
y=y^{\prime}+\frac{\Lambda}{9}\left(-t^{\prime 2}-\frac{y^{\prime 2}}{2}+\frac{\left(x^{\prime 2}+z^{\prime 2}\right)}{4}\right) y^{\prime} \\
z=z^{\prime}+\frac{\Lambda}{9}\left(-t^{\prime 2}-\frac{z^{\prime 2}}{2}+\frac{\left(x^{\prime 2}+y^{\prime 2}\right)}{4}\right) z^{\prime} \\
t=t^{\prime}-\frac{\Lambda}{18}\left(t^{\prime 2}+r^{\prime 2}\right) t^{\prime}
\end{gathered}
$$

transforms the metric into a static solution at order $\Lambda$

$$
d s^{2}=\left[1-\frac{\Lambda}{3} r^{\prime 2}\right] d t^{\prime 2}-\left[1-\frac{\Lambda}{6}\left(r^{\prime 2}+3 x_{i}^{\prime 2}\right)\right] d x_{i}^{\prime 2} .
$$

This metric has a $Z_{3}$ symmetry only. We are still in the Lorenz gauge.

Under the following additional change

$$
\begin{gathered}
x^{\prime}=x^{\prime \prime}+\frac{\Lambda}{12} x^{\prime \prime 3}, \quad y^{\prime}=y^{\prime \prime}+\frac{\Lambda}{12} y^{\prime \prime 3}, \quad z^{\prime}=z^{\prime \prime}+\frac{\Lambda}{12} z^{\prime \prime 3}, \\
t^{\prime}=t^{\prime \prime}
\end{gathered}
$$

the metric becomes

$$
d s^{2}=\left[1-\frac{\Lambda}{3} r^{\prime \prime 2}\right] d t^{\prime \prime 2}-\left[1-\frac{\Lambda}{6} r^{\prime \prime 2}\right]\left(d r^{\prime \prime 2}+r^{\prime \prime 2} d \Omega^{2}\right),
$$

which is not in the Lorenz gauge anymore. Yet another change

leads to

$$
r^{\prime \prime}=\hat{r}+\frac{\Lambda}{12} \hat{r}^{3} \quad t^{\prime \prime}=\hat{t}
$$

$$
d s^{2}=\left[1-\frac{\Lambda}{3} \hat{r}^{2}\right] d \hat{t}^{2}-\left[1+\frac{\Lambda}{3} \hat{r}^{2}\right] d \hat{r}^{2}+\hat{r}^{2} d \Omega^{2} .
$$

This is the linearized Schwarzschild-de Sitter metric.

Now we know in which coordinates we were when we solved the linearized Einstein equation with a cosmological constant in Lorenz gauge. A series of elementary coordinate transformations brought our solution to a linearized version of the Schwarzschild-de Sitter metric (expanded to first order in $\Lambda$ ). Thanks to Birkhoff's theorem[14], we know that this metric is unique.

The SdS coordinates are useful for problems with spherical symmetry, such as objects falling onto each other. They do not admit a Newtonian limit if $\Lambda \neq 0$, i.e.

$$
d s^{2} \neq(1+2 \Phi) d t^{2}-(1-2 \Phi) d x^{2}
$$

because in addition to the scalar potential $\Phi$ (which itself has a correction of $O(\Lambda)$ ) there is a tensor potential $\tau_{i j} \sim \Lambda$. However it is still true that if a massive source of mass $M$ is introduced via

$$
T_{00}=M \delta(\hat{r})
$$

the equation of motion for a non-relativistic body are governed by $\Phi$ alone

$$
\ddot{\hat{r}}=-\frac{G M}{\hat{r}}+O(\Lambda)
$$

Solutions are periodic in coordinates $\hat{r}, \hat{t}$ (up to $O(\Lambda)$ ) and the wave equation will lead to harmonic GW in these coordinates up to corrections of $O(\Lambda)$.

However, the coordinates where the metric is Schwarzschild-de Sitter centered in a remote black hole are not 'useful' for cosmology simply because we do not perform measurements here using these. But we know how to go from these SdS coordinated to the ones (FRW) where cosmological measuments are made. Of course none of these subtleties occur if $\Lambda=0$. 


\section{GRAVITATIONAL WAVES IN COSMOLOGICAL COORDINATES}

Let us go back to Lorenz gauge. Recall that we want $h_{\mu v}=h_{\mu \nu}^{\Lambda}+h_{\mu \nu}^{W}$ and that $h_{\mu \nu}^{W}$ should fulfill, if the term $\Lambda h^{W}$ is neglected,

$$
\tilde{h}_{\mu}^{W \mu}=0, \quad \partial_{\mu} \tilde{h}_{v}^{W \mu}=0, \quad \square \tilde{h}_{\mu v}^{W}=0
$$

while $h_{\mu \nu}^{\Lambda}$ should fulfill if $\Lambda h^{\Lambda}$ is neglected

$$
\tilde{h}_{\mu}^{\Lambda \mu}=0, \quad \partial_{\mu} \tilde{h}_{v}^{\Lambda \mu}=0, \quad \square \tilde{h}_{\mu v}^{\Lambda}=-2 \Lambda \eta_{\mu v}
$$

The general solution at lowest order (written for $h_{\mu v}$ ) will be

$$
h_{\mu v}=h_{\mu v}^{\Lambda}+h_{\mu v}^{W}=\frac{\Lambda}{9}\left(x_{\mu} x_{v}+2 \eta_{\mu v} x^{2}\right)+E_{\mu v}^{W} \cos k x+D_{\mu v}^{W} \sin k x
$$

where $E_{\mu \nu}$ and $D_{\mu \nu}$ are polarization tensors having vanishing traces $E^{W}=D^{W}=0$ and obeying the condition $k_{\mu} E_{v}^{\mu W}=k_{\mu} D_{v}^{\mu W}=0$ with $k^{2}=0$.

It is possible to derive the full solution including $\Lambda h_{\mu v}$ terms but we shall not consider it here as the modifications are unrealistically small to be seen, but they have some interesting aspects nevertheless. The interested reader can see [9] for details.

This coordinate system is easily related to SdS coordinates. These coordinates are well suited to describe problems with spherical symmetry ( $\hat{r}=0$ is a 'special' point) such as the solar system or collapse on to a black hole but these coordinates are not the ones where we observe the (expanding) universe. When the spherical symmetry is lost (away from the source), we have to match to suitable coordinates, physical to the observer.

We have to transform now the solutions found in SdS-like coordinates to FRW coordinates. Let us show here for simplicity just how the lowest order solution (i.e. the one obtained neglecting $\Lambda h_{\mu \nu}^{W}$ terms) looks once transformed. A plane wave propagating in the $\hat{z}$ direction transforms into

$$
\begin{aligned}
& h_{\mu v}^{W_{F R W}}=\left(\begin{array}{cccc}
0 & 0 & 0 & 0 \\
0 & E_{11}\left(1+2 \sqrt{\frac{\Lambda}{3}} T\right) & E_{12}\left(1+2 \sqrt{\frac{\Lambda}{3}} T\right) & 0 \\
0 & E_{12}\left(1+2 \sqrt{\frac{\Lambda}{3}} T\right) & -E_{11}\left(1+2 \sqrt{\frac{\Lambda}{3}} T\right) & 0 \\
0 & 0 & 0 & 0
\end{array}\right) \times \\
& \cos \left(w(T-Z)+w \sqrt{\frac{\Lambda}{3}}\left(\frac{Z^{2}}{2}-T Z\right)+\mathscr{O}(\Lambda)\right)+\mathscr{O}(\Lambda) \\
& +\left(\begin{array}{cccc}
0 & 0 \\
0 & D_{11}\left(1+2 \sqrt{\frac{\Lambda}{3}} T\right) & D_{12}\left(1+2 \sqrt{\frac{\Lambda}{3}} T\right) & 0 \\
0 & D_{12}\left(1+2 \sqrt{\frac{\Lambda}{3}} T\right) & -D_{11}\left(1+2 \sqrt{\frac{\Lambda}{3}} T\right) & 0 \\
0 & 0 & 0 & 0
\end{array}\right) \times \\
& \sin \left(w(T-Z)+w \sqrt{\frac{\Lambda}{3}}\left(\frac{Z^{2}}{2}-T Z\right)+\mathscr{O}(\Lambda)\right)+\mathscr{O}(\Lambda)
\end{aligned}
$$

The maxima of the wave will be reached when

$$
\begin{gathered}
w(T-Z)+w \sqrt{\frac{\Lambda}{3}}\left(\frac{Z^{2}}{2}-T Z\right)=n \pi \\
Z_{\max }(n, T) \simeq T-\frac{n \pi}{w}-\frac{T^{2}}{2} \sqrt{\frac{\Lambda}{3}}+\frac{n^{2} \pi^{2}}{2 w^{2}} \sqrt{\frac{\Lambda}{3}}
\end{gathered}
$$


The phase velocity of the wave is

$$
v_{p}(T) \equiv \frac{d Z_{\max }}{d T}=1-T \sqrt{\frac{\Lambda}{3}}+\mathscr{O}(\Lambda)
$$

In comoving coordinates the phase velocity is smaller than 1 . This does not mean that the waves slow down. We can calculate the velocity in 'ruler' distance.

$$
-d l^{2}=-\left(1+2 T \sqrt{\frac{\Lambda}{3}}\right) d Z^{2}, \quad \frac{d l}{d T}=\frac{d}{d T}\left[\left(1+T \sqrt{\frac{\Lambda}{3}}\right) d Z_{\max }\right] \simeq 1
$$

Notice that the modifications due to the cosmological constant are not of order $\Lambda$ as a naive consideration of the linearized field equation (obeyed by the GW) would lead us to believe. As we discussed in much detail, this linearized equation in the Lorenz gauge is in an essential way related to SdS coordinates. The effect of the coordinate change to the cosmological FRW coordinates is actually an effect of order $\sqrt{\Lambda}$ and therefore the magnitude of the change can be very different.

Several physical magnitudes appear in the modified expression for the waves; in particular $\Lambda$. Its effects are shown in the accompanying figure and the most relevant question is: are these changes detectable?

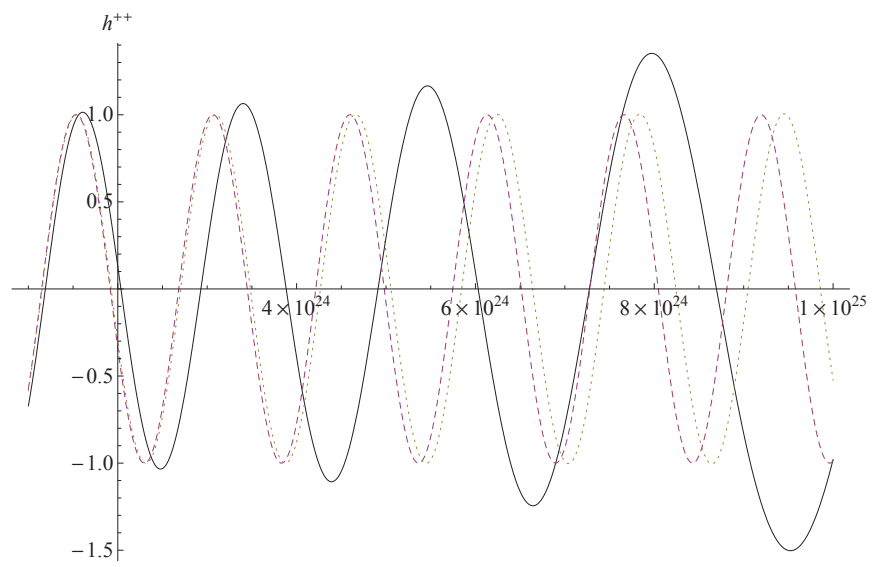

FIGURE 1. Dependence of the amplitude and wave-length on $Z$ (expressed in meters) for a constant value of $T$ and for different values of $\Lambda$. Dashed line: $\Lambda=0$, dotted line: $\Lambda=10^{-52} \mathrm{~m}^{-2}$, solid line: $\Lambda=10^{-51} \mathrm{~m}^{-2}$. Waves with $10^{3} \mathrm{~Hz}<w<10^{-10} \mathrm{~Hz}$ cannot be practically plotted in the relevant $Z$-range. Here $w=4 \cdot 10^{-16} \mathrm{~Hz}$

\section{TIMING RESIDUALS IN PULSAR ARRAYS}

Pulsars are very stable clocks with periods ranging from milliseconds up to about 10 seconds. About 600 pulsars are known. The average period is $0.65 \mathrm{~s}$. Fast rotating pulsars can be more regular than atomic clocks and disruptions of the order of $1 \mu$ s can be measured on Earth.

The passage of a gravitational wave disrupts this array of clocks and indeed PTA's may provide the first direct evidence of gravitational waves in $<10$ years. The idea behind the PTA collaborations is to detect the correlated disruption of the periods measured for a significant number of pulsars due to the passing of a gravitational wave through the system $[15,16,17,18]$. PTA are suitable detectors for low frequency GW, i.e. for the range $10^{-9} \mathrm{~Hz}$ to $\leq w \leq 10^{-7} \mathrm{~Hz}$ [15] and the signal is expected to follow a power law [16, 19]. A key problem in making predictions is modeling in a realistic way the wave functions produced in the different sources, in particular the value of the amplitude of the metric perturbation $h$ is a free parameter in principle. Some bounds in the range of $10^{-17} \leq h \leq 10^{-15}$ have been set already [19].

Rough estimates from the expressions in the previous section indicated that corrections of $O(\sqrt{\Lambda})$ can be relevant for pulsar timing arrays (typically situated at $L \sim 1 \mathrm{kpc}$ ) being disturbed by extragalactic binary black hole systems (typically at $l \sim 100 \mathrm{Mpc}$ or more). 
If $\phi_{0}(t)$ is the field phase of the pulsar, the pulsed emission measured with an Earth-based radio telescope will be

$$
\phi(t)=\phi_{0}\left(t-\frac{L}{c}-\tau_{E+S}(t)-\tau_{G W}(t)\right)
$$

where $\tau_{E+S}$ are local corrections due to the movement of the Earth and solar system and $\tau_{G W}(t)$ is the shift due to the passage of a GW.

Let us consider the observational set-up shown in the figure The field phase shift due to the GW will be approxi-

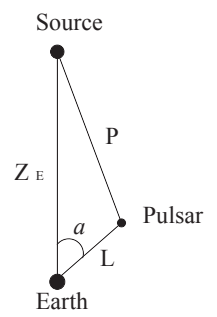

mately given by[20]

$$
\tau_{G W}(t)=-\frac{1}{2} \hat{n}^{i} \hat{n}^{j} H_{i j}(t)
$$

where

$$
H_{i j}(t)=L \int_{-1}^{0} d x h_{i j}(t+L x, \vec{P}+L(1+x) \hat{n})
$$

and $\vec{P}$ is the pulsar location, $\hat{n}=(-\sin \alpha, 0, \cos \alpha)$ and $Z_{E}=c z \sqrt{\frac{3}{\Lambda}}(z$ is the redshift). Here and in what follows we do not take matter into account, that is $\Omega_{M}=0$. We shall return to this point later.

It is interesting to note that this expression (which includes the leading correction anyway) is valid only if the time components of the perturbed metric are all zero. This is so at order $\sqrt{\Lambda}$, which by far dominates, but ceases to be true when one considers the $\mathscr{O}(\Lambda)$ terms indicated in (33). See [9] for the complete expressions.

To keep things as simple as possible let us assume that a remote merging of galaxies eventually leads to the merging of their central black holes. Characteristically, the two black holes have very different masses and therefore we can think of one object orbiting around the most massive one, and eventually collapsin onto it. The problem is therefore a keplerian one in essence, with approximate spherical symmetry. The two spiraling black holes produce GW with a characteristic time of emission that is of the order of one to several years and the period of the signal ranges from days to months. The coordinates where the emission is just a collection of a few harmonics will of course be SdS coordinates. Consider the simplest possible case (just one harmonic):

$$
h_{\mu v}^{S d S}=\frac{1}{r}\left(E_{\mu v} \cos [w(t-r)]+D_{\mu v} \sin [w(t-r)]\right)+O(\Lambda)
$$

When we say days or months we have to be definite about which clock we are talking about; namely in which coordinate system $t$ and $r$ are measured in the previous equation. It should be obvious that they refer to the coordinates associated to the supermassive black hole (located at $r=0$ ). It is only in those coordinates that the emission of GW is periodic. (Of course it is not exactly periodic as the smallest black hole loses energy and eventually collapses and the emission of the GW cannot be really attributed to the point $r=0$, but this does not change the essence of the argument because the uncertainty in the point of emission is much smaller than other magnitudes relevant for the discussion.) In conclusion, the previous equation is the distortion to the metric produced by GW in SdS coordinates.

If the metric would be exactly Schwarzchild, that is without any cosmological constant at all, the metric would asymptotically become Minkowski and these coordenates are roughly speaking also the ones of a remote observer (remember that we have neglected the presence of matter completely). However, this is certainly not the case if $\Lambda \neq 0$ because then the metric is not asymptotically flat. Then it is clear that a terrestrial observer is not using the coordinates $r$ and $t$ but rather describes their observations of the universe (in particular the distant place where the black hole merging took place) using FRW coordinates $R$ and $T$. 
As we learned in the previous sections then the GW becomes in FRW coordinates

$$
\begin{aligned}
& h_{\mu v}^{F R W}=\frac{E_{\mu v}}{R}\left(1+\sqrt{\frac{\Lambda}{3}} T\right) \cos \left[w(T-R)+w \sqrt{\frac{\Lambda}{3}}\left(\frac{R^{2}}{2}-T R\right)\right] \\
& +\frac{D_{\mu v}}{R}\left(1+\sqrt{\frac{\Lambda}{3}} T\right) \sin \left[w(T-R)+w \sqrt{\frac{\Lambda}{3}}\left(\frac{R^{2}}{2}-T R\right)\right]+O(\Lambda) .
\end{aligned}
$$

As we discussed in detail in the previous section there are corrections of order $\Lambda$ to the expression for the waves in $\operatorname{SdS}$ coordinates, but after changing to FRW coordinates, these corrections will still be of order $\Lambda$. It is really the change from SdS to FRW coordinates that matters and introduces corrections of order $\sqrt{\Lambda}$.

From the pulsar to the Earth the electromagnetic signal follows the trajectory given by the line of sight $\vec{R}(x)=$ $\vec{P}+L(1+x) \hat{n}$. $L$ is the comoving distance (replacing it by the ruler distance makes no significant differences).

$$
R(x)=\sqrt{Z_{E}^{2}+2 x L Z_{E} \cos \alpha+x^{2} L^{2}} \simeq Z_{E}+x L \cos \alpha,
$$

In the usual treatment, the cosmological constant is neglected and the effect of $\Lambda$ would taken into account only through the redshift $w \rightarrow w_{\text {eff }}=w /(1+z)$. The important question is of course whether $\Lambda$ is really relevant after all.

\section{OBSERVING COSMOLOGICAL CONSTANT EFFECTS IN GRAVITATIONAL WAVES}

Let us define

$$
\begin{aligned}
& \Theta\left(x, T_{E}, L, \alpha, \beta, Z_{E}, w, \Lambda\right) \equiv w\left(T_{E}+\frac{L}{c} x-\frac{Z_{E}}{c}-x \frac{L}{c} \cos \alpha\right) \\
& +w \sqrt{\frac{\Lambda}{3}}\left(\frac{\left(\frac{Z_{E}}{c}+x \frac{L}{c} \cos \alpha\right)^{2}}{2}-\left(T_{E}+\frac{L}{c} x\right)\left(\frac{Z_{E}}{c}+x \frac{L}{c} \cos \alpha\right)\right) .
\end{aligned}
$$

Then

$$
\begin{aligned}
H \equiv \tau_{G W}\left(T_{E}\right) & =-\frac{1}{2} \frac{L \varepsilon}{c}\left(\sin ^{2} \alpha \cos ^{2} \beta+2 \sin \alpha \sin \beta \cos ^{2} \beta-\sin ^{2} \alpha \sin ^{2} \beta\right) \\
& \times \int_{-1}^{0} d x \frac{1}{\left(Z_{E}+x L \cos \alpha\right)}\left(1+\sqrt{\frac{\Lambda}{3}}\left(T_{E}+\frac{L}{c} x\right)\right)(\cos \Theta+\sin \Theta),
\end{aligned}
$$

where $\varepsilon$ is a characteristic GW amplitude. All the variables have already been defined except $\beta$ that corresponds to the azimuthal angle of the pulsar referred to the plane perpendicular to the line Earth-source.

The first indication that the cosmological constant matters comes from considering the theoretical dependence of the signal on the angle $\alpha$ subtended by the source and the pulsar, the Earth being the vertex. This is shown in the figure where an enhancement is found at low angles (at least for the values selected for the astrophysical parameters)

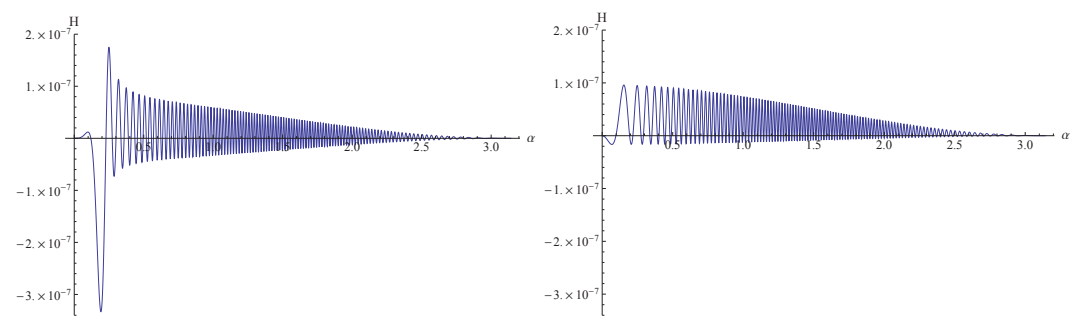

FIGURE 2. On the left the raw timing residual for $\Lambda=10^{-35} s^{-2}$ as a function of the angle $\alpha$ subtended by the source and the measured pulsar as seen from the observer. On the right the same timing residual for $\Lambda=0$. In both cases we take $\varepsilon=1.2 \times 10^{9} \mathrm{~m}$, $L=10^{19} \mathrm{~m}$ and $T_{E}=\frac{Z_{E}}{c} S$ for $Z_{E}=3 \times 10^{24} \mathrm{~m}$; with these values $|h| \sim \frac{\varepsilon}{R} \sim 10^{-15}$ which is within the expected accuracy of PTA. Similar results are obtained for other close values of $T_{E}$

Let us now define the following statistical significance

$$
\sigma=\sqrt{\frac{1}{N_{p} N_{t}} \sum_{i=1}^{N_{p}} \sum_{j=1}^{N_{t}}\left(\frac{H\left(T_{E}^{i, j}, L_{i}, \alpha_{i}, \beta_{i}, Z_{E}, w, \varepsilon, \Lambda\right)}{\sigma_{t}}\right)^{2}}
$$


where $\sigma_{t}$ is the accuracy with which we are able to measure the pulsar signal period. We take $\sigma_{t} \simeq 10^{-6} s$ (this is the average of the best measured pulsars included in the International PTA Project [17]).

We assume an observation time of 3 years, starting at the time the signal is $10^{16} \mathrm{~s}$ old (time of arrival at our Galaxy) and observations every 11 days $\left(N_{t}=101\right) ; 10^{16} s \leq T_{E} \leq 1.00000001 \times 10^{16} \mathrm{~s}$. The coalescence times of supermassive black holes is taken to be $O\left(10^{7}\right) \mathrm{s}$; that is about one year (much shorter time scale than the time of arrival of the perturbation to the local system).

The galactic latitude and longitude of each pulsar are transformed to $(\alpha, \beta)$, where $\alpha$ is the angular separation between the line Earth-GW source and the line Earth-pulsar.

We plot $\sigma(\alpha)$ using a set of 5 fixed pulsars supposed to be exactly at the same angular separation from a source the position of which we vary. This could be done for any set of five pulsars. The position of the peak does not depend on $L_{i}$ and $\beta_{i}$. We use the pulsars which are all close to each other at a distance $L \sim 10^{20} \mathrm{~m}$ :

TABLE 1.

\begin{tabular}{|c|}
\hline Pulsars from the ATNF Catalogue \\
\hline J0024-7204E \\
J0024-7204D \\
J0024-7204M \\
J0024-7204G \\
J0024-7204I \\
\hline
\end{tabular}

The results are summarized in the following figures:
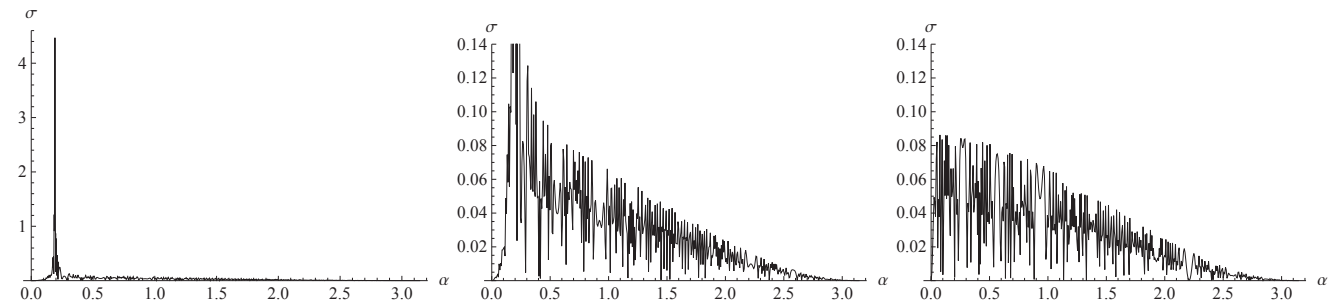

FIGURE 3. $\sigma(\alpha)$ for $\Lambda=10^{-35} s^{-2}$ (left). Zoom on the lower values for $\Lambda=10^{-35} s^{-2}$ (middle) with the prominent spike removed (notice the very different vertical scale, and comparison to the $\Lambda=0$ case (right).

As is obvious from the figure, the signal shows a remarkable similarity between the cases $\Lambda=0$ and $\Lambda \neq 0$ except for the very prominent spike that the case shown for $\Lambda=10^{-35} s^{-2}$, the currently preferred value for $\Lambda$, at a relatively low angle.

Now take a list of observed pulsars well distributed in the galaxy. The angles $(\alpha, \beta)$ are calculated for all of them considering two hypothetical sources of GW. One located at galactic coordinates $\theta_{S 1}=300^{\circ}, \phi_{S 1}=-35^{\circ}$ and another at $\theta_{S 2}=4^{\circ}, \phi_{S 2}=10^{\circ}$.

We order them from the lowest $\alpha$ to the largest. We group them in sets of five pulsars. We consider 27 sets of 5 pulsars; that is a list of 135 pulsars. For each set we calculate the significance

$$
\sigma_{k}=\sqrt{\frac{1}{5 \cdot 101} \sum_{i=1}^{5_{k}} \sum_{j=1}^{101}\left(\frac{H\left(T_{E}^{i, j}, L_{i}, \alpha_{i}, \beta_{i}, 10^{24}, 10^{-8}, 1.2 \times 10^{9}, 10^{-35}\right)}{10^{-7}}\right)^{2}}
$$

and plot it as a function of the average angle of the set, $\bar{\alpha}_{k}=\sum_{i=1}^{5_{k}} \frac{\alpha_{i}}{5}$ with $1 \leq k \leq 27$. We think that the figures speak by themselves. It is clear that PTA observations that aim at observing the 'normal' GW spectrum should absolutely see the 'abnormal' enhancement at a given value of the angle.

\section{WHY THIS ENHANCEMENT?}

In order to understand why this effect comes about let us examine the behaviour of the differential timing residual as we move along the line of sight. The window in the angular variable corresponds to a 'valley' where the phase is (nearly) stationary. At this value of the angular variable $\alpha$ is where the enhancement takes place. 


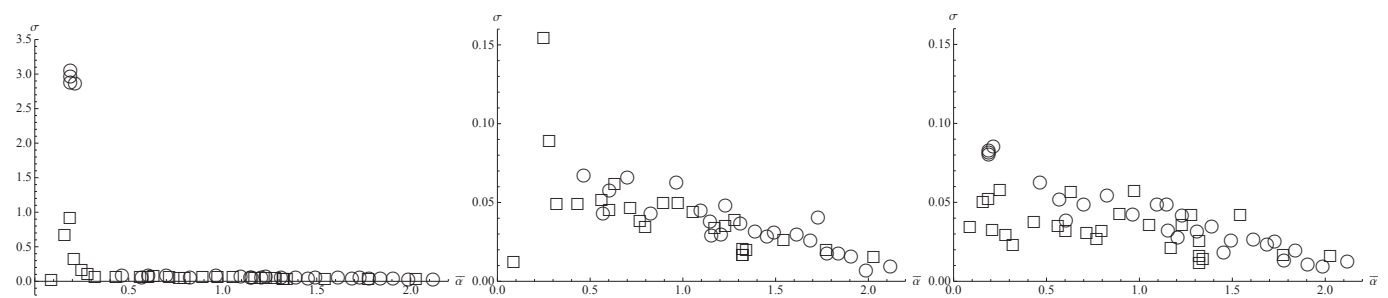

FIGURE 4. Plot of $\sigma_{k}\left(\bar{\alpha}_{k}\right), k=1,27 . \Lambda=10^{-35} s^{-2}$. Circles correspond to Source 1 and squares to Source 2. Full range is showed on the left, zoom on the lower values for $\Lambda=10^{-35} s^{-2}$ is shown in the middle (again the spike is removed -vertical scale is different) and comparison to $\Lambda=0$ is show on the right figure, respectively.

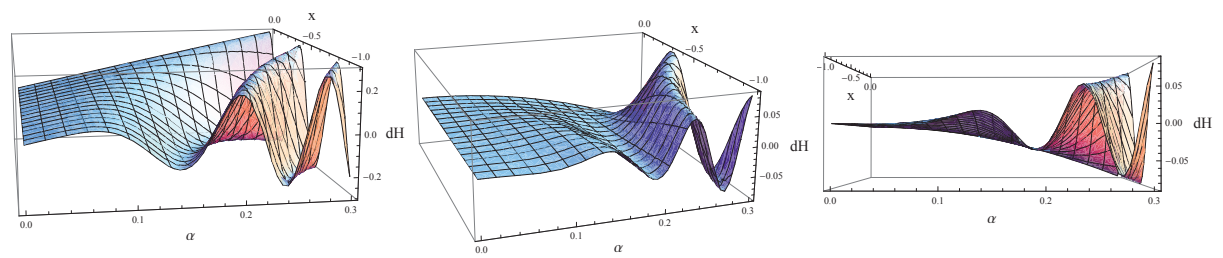

FIGURE 5. Evolution of the phase of the GW along the line of sight to the pulsar for different angles. Left figure $\Lambda=0$. Middle: $\Lambda=10^{-35} s^{-2}$. Right: same as the previous one but rotated so as to show the 'valley' of stationary phase, which is absent without the cosmological constant.

Among all the dependencies, and when the distance to the source is well known, the most relevant appears to be the one related to the value $\Lambda$. The position of the peak depends strongly on the value of $\Lambda$. It moves towards the central values of the angle for larger values of the cosmological constant.

The position of the 'valley' (and therefore $\Lambda$ ) can be found analytically in two ways

1. Looking for the stationary phase condition

2. Examining the behaviour of the Fresnel functions and prefactors obtained after integration

The prefactor becomes quite large for a specific value of the parameters involved. This particular value renders the Fresnel function close to zero and the product is a number close to 2. Away from this point the net result is small.

Using the series expansion of the Fresnel functions at first order we are able to obtain an approximate analytical expression for the relation $\Lambda(\alpha)$

$$
\Lambda(\alpha)=\frac{12 c^{2} \sin ^{4}\left(\frac{\alpha}{2}\right)}{\left(\left(c T_{E}-Z_{E}\right) \cos \alpha+Z_{E}\right)^{2}} \simeq \frac{12 c^{2} \sin ^{4}\left(\frac{\alpha}{2}\right)}{Z_{E}^{2}},
$$

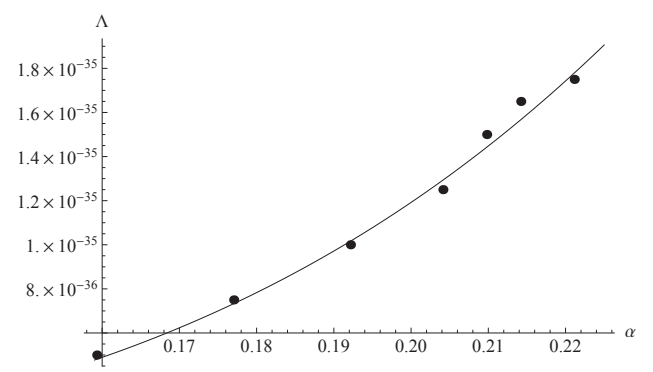

FIGURE 6. $\Lambda(\alpha)$ obtained numerically from the positions of the peaks in the $\sigma(\alpha)$ plots for different values of the cosmological constant (dots) and obtained analytically from an approximation of the Fresnel functions involved in the timing residual (line). 
However it is possibly more enlightening to understand the effect via the first explanation (which of course is mathematically equivalent to the second, but clearer from a physical viewpoint). For the sake of this discussion consider a scalar wave with the same arguments as (42), parametrized along a null geodesic using (we set here again $c=1$ and choose the initial phase to be convenient for our discussion)

$$
t=T+x L, \quad r=R+x L \cos \alpha, \quad-1<x<0,
$$

i.e.

$$
\phi=\frac{1}{R+x L \cos \alpha} \cos \left[w x L(1-\cos \alpha)+w \sqrt{\frac{\Lambda}{3}}\left(\frac{1}{2}(R+x L \cos \alpha)^{2}-(R+x L \cos \alpha)(T+x L)\right)\right] .
$$

If we take the derivative of the argument with respect to $\mathrm{x}$ and keep only the terms that really matter for the relevant values of the parameters, we get

$$
\frac{d \phi}{d x} \simeq w\left(L(1-\cos \alpha)+\sqrt{\frac{\Lambda}{3}}(-L T \cos \alpha-L R(1-\cos \alpha))\right) .
$$

As just stated, in the derivative we have neglected some terms proportional to $x$ that are very small. Now using that $T \simeq R$ to a very good precision, it can be shown this expression has a zero at the value of $\alpha$ corresponding to

$$
\sin ^{4} \alpha \simeq \frac{4}{3} R^{2} \Lambda
$$

that for small values of $\alpha$ agrees with (48).

The enhancement's angular position corresponds very approximately to a stationary line of the wave. The value of the phase of the wave remains practically constant (with a wave amplitude different from zero) along the straight line from the pulsar to the Earth for that particular value of $\alpha$. So the integrated timing residual is maximized for this angle.

The limit $\Lambda=0$ also has such stationary phase, it obviously corresponds to $\alpha=0$ where the light signal from the pulsar and the GW travel in phase towards us, but the relevant point is that the angle $\alpha$ where the enhancement is observed gives a very direct determination of the cosmological constant, a remarkable property that was not noticed before. As we see the only unknowns are the distance $R$ and $\Lambda$ itself, which is remarkable. In particular the effect is independent of the frequency, and also of $L$, the distance to the given pulsar used as a clock. Of course if we trust that $\Lambda$ is a universal constant value this expression can be used to obtain $R$, the distance from the Earth to the source of GW.

\section{A CRITICAL LOOK}

Although the results presented are quite interesting there are several points on which they are manifestly incomplete and therefore the conclusions one may draw can be criticized.

1. The wave front used is not realistic. Indeed, we have assumed a periodic wave with only one harmonic. However, it would be quite straightforward to extend the analysis to the different Fourier components of a given signal and the essence of the discussion would be exactly the same. Because the effect is relatively insensitive to the frequency[10] and depends mostly on $Z_{E}$ and $\Lambda$ we would not expect substantial changes in the conclusions. This is surely the least relevant of the possible objections.

2. The time dependence of the signal will average out the effect. We have sometimes been asked why the effect is not averaged out. This objection seems to arise from a misunderstanding of formula (45). It is true that the period of observation is typically much longer than the typical period of the black hole spiraling. However as is obvious from the explanation provided in the previous section, the frequency itself plays no role in the construction of the 'valley' corresponding to the stationary phase condition. The only time that plays a role at all is $T_{E}$, and indeed the signal would average out after a time $>>T_{E}$, but this is a very long time, many orders of magnitude longer than the observational period.

3. The matter distribution in the galaxy will perturb the signal and finally wash it out. This is a valid concern as local distortions of the background metric certainly influence to some extent the propagation of GW. Indeed although globally $\Lambda$ contributes more to the matter-energy budget of the universe, in the Galaxy the balance is obviously 
reversed (in fact it will be dominated by the dark matter component of the halo). However, this and similar effects arise from non-linear terms in the equation governing GW propagation. In fact they are similar in effects, but locally larger, than the term $\Lambda h_{\mu \nu}^{W}$ that was neglected in the discussion. But of course they are relevant only in the vicinity of the Galaxy. When the GW arrive at the Galaxy, anharmonicity is already well developed and changes with respect to the $\Lambda=0$ case noticeable. Although the fine details remain to be worked out, it seems extremely unlikely that these effects could be really significant.

There are two more effects that would need to be taken into account due to the matter distribution in the Galaxy. First of all, the time for the pulsar(s) signal to reach the Earth in the absence of the GW is not exactly $L / c$ as there are local perturbations on light propagation. However, these are very slowly varying with time - except for the peculiar motion of the Earth and Sun, separately taken into account. This is therefore in any case an additional term that does not really change the effect.

A last ingredient to be taken into account is to consider the modifications brounght about by the matter distribution in the Galaxy in the precise relation between the cosmological time and the observer's time defined by a clock on the Earth. It does not seem that this can have any effect at all in the kind of signal predicted.

4. Background noise will make the signal undetectable. In the universe there is not a single source of gravitational waves of this kind and we should expect that the PTA detect many such events [21]. Unfolding the signal is of course non-trivial but it does not seem to be worse in our proposal than in the usual case. In practice this is a serious difficulty in all GW detectors, not just PTA. In fact the predicted enhancement at a given angle should facilitate things.

5. The matter density has been neglected. This is by far the most serious shortcoming of our proposal. So far we have not taken into account the fact that $\Omega_{M}$ in non-zero in the universe and we have not taken into account the effects due to the masses of the coalescent black holes. Both effects should definitely be taken into account.

\section{CONCLUSION AND OUTLOOK}

In this presentation we have summarized our recent joint work with J. Bernabeu and D. Puigdomènech on the issue of GW in de Sitter space-time. We study a very definite problem: how a GW produced in the merging of two black holes propagates in de Sitter space-time and how is detected. Conventionally, the effect of the cosmological constant is included via the usual redshift in the wave frequency. We criticize this simple approach and see that there is a lot more to it.

In order to understand the role of $\Lambda$ in the propagation of GW using a linearized approximation seems essential. This linearization procedure is of course the common one to treat small perturbations around Minkowski space-time (in a sense, this is necessary in order to define a gravitational wave and also to keep track of the $\mathscr{O}(\Lambda)$ corrections. Another line of work studies the small perturbations around a FRW metric corresponding to primordial GW. It should be clear to the reader that this is not what interests us here. Here the problem to study is completely different and in order to keep track the different orders in $\Lambda$ it is useful to linearize both the background and the GW perturbation.

To understand the propagation of GW the different coordinate systems involved have to be thoroughly understood. As a result of the anlaysis GW that are harmonic when produced in the merging of two supermassive black holes turn out to be anharmonic in cosmological coordinates, the ones where we observe.

In fact the modifications appear to be very relevant for PTA. There is a dramatic enhancement for a given value of the angle $\alpha$ subtended by the source of the GW and the corresponding pulsar and that depends strongly on the value of $\Lambda$.

This work is still very preliminary, for instance the fact that $\Omega_{M} \neq 0$ has not been considered yet. But the possibility that GW can be used in a not too distant future to perform a 'local' measurement of $\Lambda$ seems fascinanting and promising.

\section{ACKNOWLEDGEMENTS}

It is a pleasure to thank my collaborators J. Bernabeu and D. Puigdomènech. The financial support from grants FPA2010-20807 (MICINN), 2009SGR502 and Consolider grant CSD2007-00042 (CPAN) is gratefully acknowledged. I would like to thank A. Andrianov, V. Andrianov, S. Afonin and all the other colleagues at the University of Saint Petersburg for organizing the II Russian-Spanish joint workshop on Particle Physics where this work was presented and 
for making it very enjoyable and fruitful. The author would also like to thank the Faculty of Physics of the Universidad Católica de Chile where these proceedings were written for their warm hospitality.

\section{REFERENCES}

1. A. G. Riess et al. [Supernova Search Team Collaboration], Astron. J. 116, 1009 (1998) [arXiv:astro-ph/9805201]. S. Perlmutter et al. [Supernova Cosmology Project Collaboration], Astrophys. J. 517, 565 (1999) [arXiv:astro-ph/9812133]. A. G. Riess, Publ. Astron. Soc. Pac. 112, 1284 (2000) [arXiv:astro-ph/0005229]. E. Linder and S. Perlmutter, Phys. World 20N12 (2007) 24; M. Kowalski et al. [Supernova Cosmology Project Collaboration], Astrophys. J. 686, 749 (2008) [arXiv:0804.4142 [astro-ph]]; W. M. Wood-Vasey et al. [ESSENCE Collaboration], Astrophys. J. 666, 694 (2007) [arXiv:astro-ph/0701041]. A. G. Riess and M. Livio, Astrophys. J. 648, 884 (2006) [arXiv:astro-ph/0601319]. A. G. Riess et al. [Supernova Search Team Collaboration], Astrophys. J. 607, 665 (2004) [arXiv:astro-ph/0402512]; D. N. Spergel et al. [WMAP Collaboration], Astrophys. J. Suppl. 170, 377 (2007) [arXiv:astro-ph/0603449]; D. J. Eisenstein et al. [SDSS Collaboration], Astrophys. J. 633, 560 (2005) [arXiv:astro-ph/0501171]; H. J. Seo and D. J. Eisenstein, Astrophys. J. 598, 720 (2003) [arXiv:astro-ph/0307460]; L. Fu et al., Astronomy \& Astrophysics 479, 9 (2008); L. Guzzo et al., Nature 451, 541 (2008).

2. M. Sereno and P. Jetzer, Phys. Rev. D 73, 063004 (2006) [arXiv:astro-ph/0602438]; M. Sereno and P. Jetzer, Phys. Rev. D 75, 064031 (2007) [arXiv: astro-ph/0703121]; A. Balaguera-Antolinez, C. G. Boehmer and M. Nowakowski, Class. Quant. Grav. 23, 485 (2006) [arXiv:gr-qc/0511057]; ibid, Int. J. Mod. Phys. D 141507 (2005) [arXiv: gr-qc/0409004]; L. Iorio, Adv. in Astronomy, Article ID 268647 (2008), doi:10.1155/2008/268647; New Astron. 14, 196 (2008) [arXiv:0808.0256 [gr-qc]]; Y. Suto, Progr. Theoretical Physics 90, 1173 (1993) [arXiv:astro-ph/9609014]; G. S. Adkins, J. McDonnell and R. N. Fell, Phys. Rev. D 75, 064011 (2007) [arXiv:gr-qc/0612146].

3. I.B. Khriplovich and A.A. Pomeransky, Int. J. Mod. Phys. D 17, 2255 (2008)[arXiv:0801.1764].

4. M. Sereno, Phys. Rev. Lett. 102, 021301 (2009)[arXiv:0807.5123]; Phys. Rev. D 77, 043004 (2008) [arXiv:0711.1802].

5. W. Rindler and M. Ishak, Phys. Rev. D 76, 043006 (2007)[arXiv:0709.2948]; M. Ishak, W. Rindler, J. Dossett, J. Moldenhauer and C. Allison, Mon. Not. R. Astron. Soc. 388, 1279 (2008) [arXiv:0710.4726]; M. Ishak, W. Rindler, Gen. Rel. Grav. 42, 2247 (2010) [arXiv:1006.0014].

6. J. Bernabeu and N. Mavromatos, private communication (2013).

7. C. Castro, Phys. Lett. B 675, 226 (2009), DOI: 10.1016/j.physletb.2009.03.079.

8. L. Iorio, The Astronomical Journal 142, 68 (2011), doi:10.1088/0004-6256/142/3/6; P. Castelo Ferreira, arXiv:1203.1844.

9. J. Bernabeu, D. Espriu, D. Puigdomènech, Phys. Rev D 84, 063523 (2011) [arXiv:1106.4511]; ibid 86, 069904(E)(2012)

10. D. Espriu, D. Puigdomènech, Astrophys. J. 764, 163 (2013)[arXiv:1209.3724]

11. J. Naf, P. Jetzer and M. Sereno, Phys. Rev. D 79,024014 (2009) [arXiv:0810.5426]

12. See, for instance: W. Rindler, Relativity: Special, General, and Cosmological (Oxford University Press (Second Edition, (2006); W. Rindler, Phys. Lett. A 245, 363 (1998); the spherically-symmetric Schwarzschild-de Sitter solution was first discussed in F. Kottler, Ann. Phys. 361, 401 (1918); the Friedmann-Lemaitre-Robertson-Walker metric was first introduced in A. Friedmann, Zeitschrift für Physik A 10, 377 (1922).

13. J. Bernabeu, C. Espinoza and N. E. Mavromatos, Phys.Rev. D81:084002 (2010)[arXiv:0910.3637].

14. G. D. Birkhoff, Relativity and Modern Physics. Cambridge, MA: Harvard University Press (1923).

15. G. Hobbs et al., AIP Conf.Proc. 983, 630 (2008), DOI: 10.1063/1.2900316; G. Hobbs, Pulsars as gravitational detectors, arXiv:1006.3969; D. R. B. Yardley et al., AIP Conf.Proc. 1357, 77 (2011), DOI: 10.1063/1.3615082.

16. G. Hobbs, Class. Quant.Grav. 25, 114032 (2008)[arXiv:0802.1309]; J.Phys.Conf.Ser. 122, 012003 (2008).

17. G. Hobbs et al., Class.Quant.Grav. 27, 084013 (2010) [arXiv:0911.5206].

18. K. J. Lee et al, Gravitational wave astronomy of single sources with a pulsar timing array, DOI: $10.1111 / \mathrm{j} .1365-$ 2966.2011.18622.x; J. M. Cordes and F. A. Jenet, Astrophys. J. 752, 54 (2012), DOI: 10.1088/0004-637X/752/1/54; F. A. Jenet and J.W. Armstrong, Phys. Rev. D83 (2011) 081301 [arXiv:1101.3759]; J.A. Ellis, F.A. Jenet and M.A. McLaughlin, Astrophys. J. 753, 96 (2012) [arXiv:1202.0808].

19. F. A. Jenet et al, Astrophys. J. 653, 1571 (2006)[astro-ph/0609013]; M.A. Bizouard et al., Class. Quant. Grav. 30220301 (2013), DOI: 10.1088/0264-9381/30/22/220301; R.M. Shannon et al. Science 342 (2013) 6156, 334 [arXiv:1310.4569]; G. Hoobs, Class. Quant. Grav. 30 (2013) 224007 [arXiv:1307.2629]; P. B. Demorest et al., Astrophys. J. 762,94 (2013) [arXiv:1201.6641].

20. See e.g. X. Deng and L. S. Finn, Mon. Not. R. Astron. Soc. , (2010) [arXiv:1008.0320]

21. V. Ravi et al., Astrophys. J. 761 (2012) 84 [arXiv:1210.3854]; X. Siemens et al. Class. Quant. Grav. 30, 224015 (2013), DOI: 10.1088/0264-9381/30/22/224015; 